Online Laboratory Investigations

\title{
Influence of Inspiration to Expiration Ratio on Cyclic Recruitment and Derecruitment of Atelectasis in a Saline Lavage Model of Acute Respiratory Distress Syndrome*
}

\author{
Stefan Boehme, $\mathrm{MD}^{1,2}$; Alexander H. Bentley, $\mathrm{MD}^{2}$; Erik K. Hartmann, $\mathrm{MD}^{2}$; Shi Chang, MD, $\mathrm{PhD}^{2,3}$; \\ Gabor Erdoes, $\mathrm{MD}^{1}$; Anatol Prinzing, MD²,4 Michael Hagmann, BSc ${ }^{5}$; James E. Baumgardner, MD, PhD; \\ Roman Ullrich, $\mathrm{MD}, \mathrm{PhD}^{1}$; Klaus Markstaller, $\mathrm{MD}, \mathrm{PhD}^{1,2}$; Matthias David, $\mathrm{MD}, \mathrm{PhD}^{2}$
}

Objective: Cyclic recruitment and derecruitment of atelectasis can occur during mechanical ventilation, especially in injured lungs. Experimentally, cyclic recruitment and derecruitment can be quantified by respiration-dependent changes in $\mathrm{PaO}_{2}\left(\Delta \mathrm{PaO}_{2}\right)$, reflecting the varying intrapulmonary shunt fraction within the respiratory cycle. This study investigated the effect of inspiration to expiration ratio upon $\triangle \mathrm{PaO}_{2}$ and Horowitz index.

Design: Prospective randomized study.

\section{*See also p. 724}

'Department of Anesthesia, General Intensive Care, and Pain Management, Medical University Vienna, Vienna, Austria.

${ }^{2}$ Department of Anesthesiology, Medical Center of the Johannes-Gutenberg University Mainz, Mainz, Germany.

${ }^{3}$ Beijing Institute of Pharmacology and Toxicology, National Beijing Center for Drug Safety Evaluation and Research, Beijing, People's Republic of China.

${ }^{4}$ Department of Cardiovascular Surgery, German Heart Center Munich, Munich, Germany.

${ }^{5}$ Center for Medical Statistics, Informatics, and Intelligent Systems, Medical University Vienna, Vienna, Austria.

${ }^{6}$ Oscillogy LLC, Folsom, PA.

This work was performed at Department of Anesthesia, General Intensive Care, and Pain Management, Medical University Vienna, Vienna, Austria; and Department of Anesthesiology, Medical Center of the JohannesGutenberg University Mainz, Mainz, Germany.

Supplemental digital content is available for this article. Direct URL citations appear in the printed text and are provided in the HTML and PDF versions of this article on the journal's website (http://journals.lww.com/ccmjournal).

Drs. Boehme, Bentley, Hartmann, Chang, and Prinzing and their institutions received support for article research from the German Research Council (Deutsche Forschungsgemeinschaft) (grant DFG Pak 415: Ma 2398/6). Dr. Ullrich served as a board member for MASIMO Inc. Irvine, CA, and received support for article research from the German Research Foundation. His institution received grant support from the German Research Council (PAK 415/Ma2398/6) and the German Research Foundation. Dr. David's institution received grant support from the German Research Council (grant DFG Pak 415: Ma 2398/6). The remaining authors have disclosed that they do not have any potential conflicts of interest.

For information regarding this article, E-mail: stefan_boehme@web.de

Copyright (C) 2015 by the Society of Critical Care Medicine and Wolters Kluwer Health, Inc. All Rights Reserved.

DOI: $10.1097 / C C M .0000000000000788$
Setting: Laboratory investigation.

Subjects: Piglets, average weight $30 \pm 2 \mathrm{~kg}$.

Interventions: At respiratory rate 6 breaths/min, end-inspiratory pressure $\left(\mathrm{P}_{\text {endinsp }}\right) 40 \mathrm{~cm} \mathrm{H} \mathrm{H}_{2} \mathrm{O}$, positive end-expiratory pressure $5 \mathrm{~cm}$ $\mathrm{H}_{2} \mathrm{O}$, and $\mathrm{FIO}_{2} 1.0$, measurements were performed at randomly set inspiration to expiration ratios during baseline healthy and mild surfactant depletion injury. Lung damage was titrated by repetitive surfactant washout to induce maximal cyclic recruitment and derecruitment as measured by multifrequency phase fluorimetry. Regional ventilation distribution was evaluated by electrical impedance tomography. Step changes in airway pressure from 5 to $40 \mathrm{~cm}$ $\mathrm{H}_{2} \mathrm{O}$ and vice versa were performed after lavage to calculate $\mathrm{PO}_{2}-$ based recruitment and derecruitment time constants (TAU).

Measurements and Main Results: In baseline healthy, cyclic recruitment and derecruitment could not be provoked, whereas in model acute respiratory distress syndrome, the highest $\Delta \mathrm{PaO}_{2}$ were routinely detected at an inspiration to expiration ratio of 1:4 (range, 52-277 torr [6.9-36.9 kPa]). Shorter expiration time reduced cyclic recruitment and derecruitment significantly $(158 \pm 85$ torr $[21.1 \pm 11.3 \mathrm{kPa}$ ] [inspiration to expiration ratio, $1: 4$ ]; $25 \pm 12$ torr [3.3 $\pm 1.6 \mathrm{kPa}$ ] [inspiration to expiration ratio, 4:1]; $p<0.0001$ ), whereas the $\mathrm{PaO}_{2} / \mathrm{FlO}_{2}$ ratio increased (267 \pm 50 [inspiration to expiration ratio, 1:4]; 424 \pm 53 [inspiration to expiration ratio, $4: 1] ; p<0.0001)$. Correspondingly, regional ventilation redistributed toward dependent lung regions $(p<0.0001)$. Recruitment was much faster (TAU: fast $1.6 \mathrm{~s}$ [78\%]; slow $9.2 \mathrm{~s}$ ) than derecruitment (TAU: fast $3.1 \mathrm{~s}$ [87\%]; slow $17.7 \mathrm{~s})(p=0.0078)$.

Conclusions: Inverse ratio ventilation minimizes cyclic recruitment and derecruitment of atelectasis in an experimental model of surfactant-depleted pigs. Time constants for recruitment and derecruitment, and regional ventilation distribution, reflect these findings and highlight the time dependency of cyclic recruitment and derecruitment. (Crit Care Med 2015; 43:e65-e74)

Key Words: acute respiratory distress syndrome; cyclic recruitment and derecruitment; inverse ratio ventilation; lung atelectasis; oxygen sensing 
A $s$ a life-saving therapy, the majority of patients with acute respiratory distress syndrome (ARDS) require mechanical ventilation to support the failing lungs, but this in turn raises the risk of ventilator-induced lung injury (VILI) (1). Reducing tidal volume (VT) improves both mortality and morbidity (2-4). On the other hand, recent studies demonstrated that patients with respiratory failure differ in heterogeneity of ventilation distribution, and in this regard, lower VT results in a higher amount of atelectasis formation and the need of individually adjusted positive end-expiratory pressure (PEEP) $(5,6)$.

Several studies investigated the effects of different ventilator settings upon recruitment or derecruitment measured by static changes in airway pressure or total lung volume (typically during a breath hold) $(7,8)$. However, these studies did not consider the dynamics of cyclic recruitment and derecruitment (c-R/D) and the consequent differences between end-inspiratory and end-expiratory pauses versus these same end-inspiratory and expiratory pressures during dynamic breathing. Approaches with higher time resolution, such as dynamic CT (dCT), can distinguish between fixed atelectatic lung areas and atelectasis formations that collapse and reopen within the respiratory cycle $(9,10)$. Furthermore, it has been demonstrated in specific lung injury models that intrapulmonary shunt fraction varies at the frequency of the respiratory rate (RR), leading to huge fluctuations in $\mathrm{PaO}_{2}\left(\Delta \mathrm{PaO}_{2}\right)$ that can be measured by an ultrafast, indwelling oxygen-sensing probe in experimental settings $(11,12)$.

C-R/D is thought to be an important contributor to lung injury. However, the impact of inspiration to expiration ratio (I:E) and inverse ratio ventilation (IRV) on c-R/D has not been previously reported. Therefore, the aim of the current study was to investigate the effect of different I:E ratios on $\Delta \mathrm{PaO}_{2}$ (primary endpoint) and Horowitz index (secondary endpoint) during mechanical ventilation. Additionally, we measured the effect of I:E ratios on regional distribution of ventilation by electrical impedance tomography (EIT) and the time constants for recruitment and derecruitment by multifrequency phase fluorimetry (MFPF).

\section{MATERIALS AND METHODS}

\section{Animal Experiments}

After the state animal care committee approval (Landesuntersuchungsamt Koblenz, Rhineland Palatinate, Germany: approval number: $23177-07 /$ G09-1-029), 10 piglets $(30 \pm 2 \mathrm{~kg}$ ) were investigated in a prospective randomized study. One animal started in the study but did not make it to the end because of premature death, and thus nine animals were finally evaluated for the effects of I:E ratio. One of these animals expired before completion of measurement of time constants at the end of the study protocol. Handling of the animals was in accordance with the National Institutes of Health guidelines.

After premedication, general anesthesia was induced and maintained with continuous infusion of propofol and fentanyl. After induction of anesthesia, central vessels were cannulated.
Details of anesthetic procedures and routine monitoring are described in the supplemental data (Supplemental Digital Content 1, http://links.lww.com/CCM/B150).

\section{Study Protocol}

After catheter placement, ventilation was set as follows to assess baseline healthy (BLH) conditions: pressure controlled ventilation $(\mathrm{PCV}), \mathrm{RR} 6$ breaths/min, end-inspiratory pressure ( $\left.\mathrm{P}_{\text {endinsp }}\right) 40 \mathrm{~cm} \mathrm{H}_{2} \mathrm{O}$, PEEP $5 \mathrm{~cm} \mathrm{H} \mathrm{H}_{2} \mathrm{O}$, and $\mathrm{FIO}_{2} 1.0$ and simultaneous measurements of MFPF-PaO ${ }_{2}$, EIT, hemodynamic, and spirometry data were performed at randomly set I:E ratios of $1: 4,1: 2,1: 1,2: 1$, and $4: 1$.

Afterward, c-R/D was induced according to a specific algorithm (Fig. 1) by repetitive washout of surfactant. The goal for titration of lavages according to this algorithm was to produce a mild injury, with not only a recruitable lung at maximally recruited ventilator settings $\left(\mathrm{PaO}_{2} / \mathrm{FIO}_{2}>450\right)$ but also a lung capable of partial collapse at minimally recruited ventilator settings $\left(\mathrm{PaO}_{2} / \mathrm{FIO}_{2}<300\right)$.

Directly prior to lung lavage, the lung was recruited during tidal ventilation in PCV mode by setting $\mathrm{P}_{\text {endinsp }} 40 \mathrm{~cm} \mathrm{H}_{2} \mathrm{O}$ and PEEP $15 \mathrm{~cm} \mathrm{H}_{2} \mathrm{O}$ for several breaths, and then the tubing was clamped in inspiration and warmed normal saline $(30 \mathrm{~mL} / \mathrm{kg})$ was administered. After 30 minutes of stabilization, the I:E settings of maximal recruitment and maximal derecruitment (defined by the Horowitz index $\left[\mathrm{PaO}_{2} / \mathrm{FIO}_{2}\right]$ with standard Clark-type electrode for blood gas analysis) were evaluated by switching through the different I:E ratios (1:4, 1:2, 1:1, 2:1, and $4: 1)$ at an RR of 6 breaths/min. If the maximally recruited lung produced a $\mathrm{PaO}_{2} / \mathrm{FIO}_{2}$ ratio greater than 450 , but at maximal collapse, the $\mathrm{PaO}_{2} / \mathrm{FIO}_{2}$ ratio was still greater than 300 , another lavage was performed. If the minimal $\mathrm{PaO}_{2} / \mathrm{FIO}_{2}$ had decreased to less than 300 , or if maximal recruitment had become compromised (maximal $\mathrm{PaO}_{2} / \mathrm{FIO}_{2}<450$ ), no further lavages were performed and the animals were stabilized for 30 minutes. During all procedures, systolic arterial pressure (SAP) was kept above $70 \mathrm{~mm} \mathrm{Hg}$ by boluses of $50 \mathrm{~mL}$ hydroxyl ethyl starch (6\%) as long as there was an adequate response (increase in SAP of $>5 \mathrm{~mm} \mathrm{Hg}$ ) to the volume bolus. Otherwise, norepinephrine was administered in steps of $0.1 \mu \mathrm{g} / \mathrm{kg} / \mathrm{min}$.

After the stabilization period, $\mathrm{c}-\mathrm{R} / \mathrm{D}$ (defined as $\Delta \mathrm{PaO}_{2}$ of $>50$ torr $[6.7 \mathrm{kPa}]$ ) was detected in all animals, and no animals were dropped from the study (Fig. 1). Measurements for lavage injury were then recorded at randomized I:E ratios $(1: 4,1: 2,1: 1,2: 1$, and 4:1) while all other ventilator settings were kept fixed, analogous to BLH. For each I:E ratio, the animals were first stabilized for 15 minutes, and then MFPF- $\mathrm{PaO}_{2}$, EIT, hemodynamic, and spirometry data were recorded for 3-5 minutes. Afterward, the I:E setting was changed according to randomization list and the procedures were repeated. The duration of this part of the protocol was 100 minutes (five I:E ratios at $20 \mathrm{~min}$ each). Subsequent to I:E variation, we performed a step change maneuver in continuous positive airway pressure (CPAP) to assess recruitment and derecruitment time constants. To assess recruitment, a CPAP of $5 \mathrm{~cm} \mathrm{H}_{2} \mathrm{O}$ was applied and step changed to $40 \mathrm{~cm} \mathrm{H}_{2} \mathrm{O}$ for at least 30 seconds, 


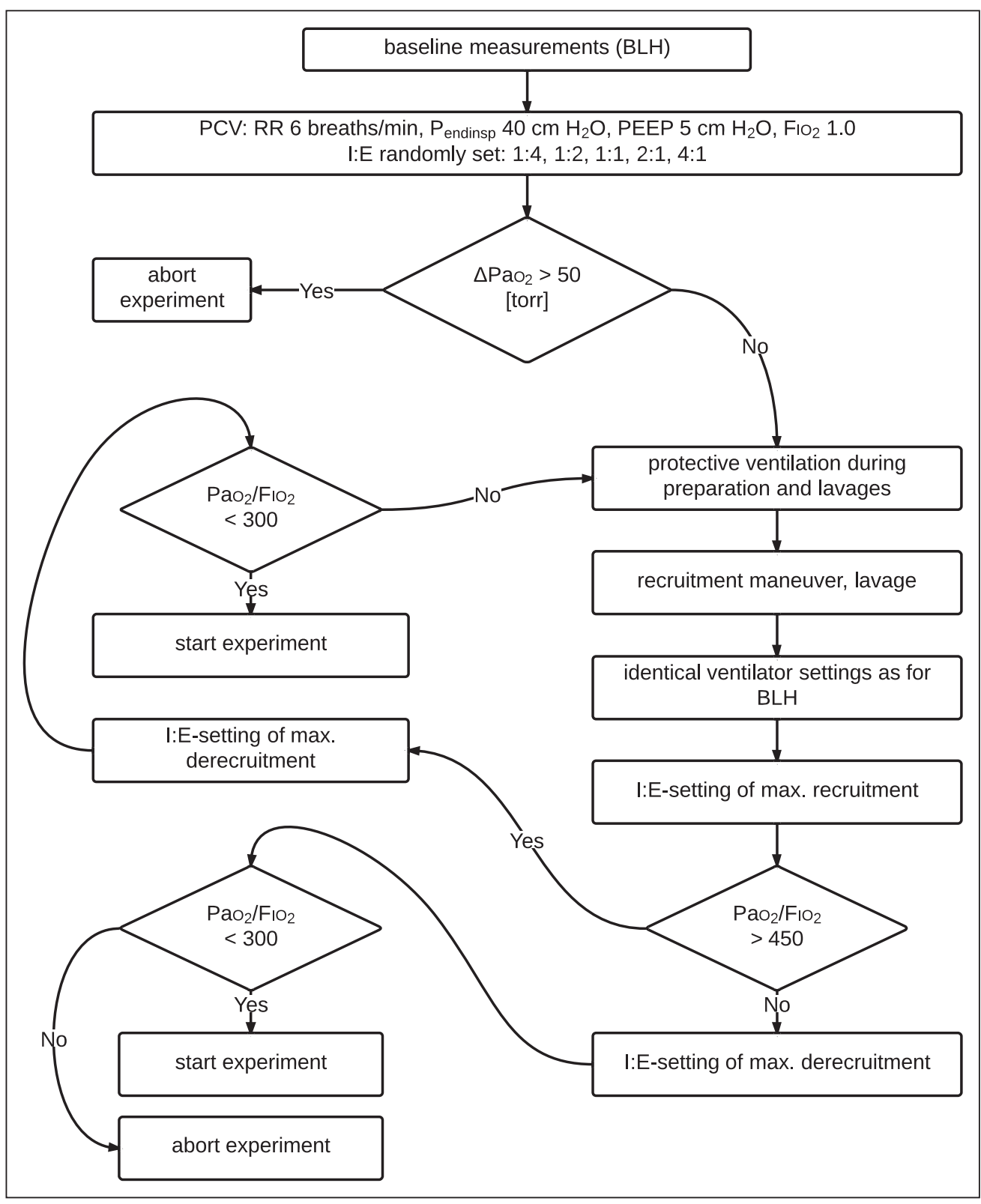

Figure 1. Study protocol and flowchart to induce cyclic recruitment and derecruitment. BLH= baseline healthy, $\mathrm{PCV}=$ pressure controlled ventilation, $\mathrm{RR}=$ respiratory rate (breaths $/ \mathrm{min}), \mathrm{P}_{\text {endinsp }}=$ end-inspiratory pressure $\left(\mathrm{cm} \mathrm{H}_{2} \mathrm{O}\right), \mathrm{PEEP}=$ positive end-expiratory pressure $\left(\mathrm{cm} \mathrm{H}_{2} \mathrm{O}\right), \mathrm{I}: \mathrm{E}=$ inspiration to expiration ratio, $\Delta \mathrm{PaO}_{2}=$ within-breath fluctuations in $\mathrm{PaO}_{2}$ (torr), $\mathrm{PaO}_{2} / \mathrm{FIO}_{2}=$ Horowitz index. attached to the MFPF measurement server unit (NeoFox, OceanOptics) via fiber optic cable and data were exported to a laptop. We used the multitemperature compensation file offered by OceanOptics and calibrated the probe prior to the experiments to air $\left(\mathrm{PO}_{2} 158\right.$ torr $[21.1 \mathrm{kPa}])$ at room temperature, in consideration of the atmospheric pressure. To confirm MFPF probe calibration standard, Clark-type electrode blood gas analyses were performed in vivo.

For each measurement point, 2 minutes of absolute $\mathrm{PaO}_{2}$ values were extracted. The resulting data were plotted over time and analyzed breath-bybreath by a Mathcad program (Mathcad v.15, PTC, Needham, MA) written by one of the authors (J.E.B.) (available on request). Mean amplitude of $\mathrm{PaO}_{2}\left(\Delta \mathrm{PaO}_{2}\right)$ was obtained by averaging the individual $\mathrm{PaO}_{2}$ amplitudes of each respiratory cycle within the measurement period. To assess the time constants for recruitment and derecruitment during the CPAP maneuver, absolute $\mathrm{PaO}_{2}$ was plotted over time and the time constants were calculated by best nonlinear fit, as described in the supplemental methods (Supplemental Digital Content 1, http://links. lww.com/CCM/B150). whereas the $\mathrm{PaO}_{2}$ time course was recorded. For derecruitment, the step change was from 40 to $5 \mathrm{~cm} \mathrm{H}_{2} \mathrm{O}$.

\section{Real-Time Arterial $\mathrm{Po}_{2}$ Measurement via MFPF}

As described in detail previously (12), an increase in atelectasis during exhalation leads to a rapid increase in shunt fraction. During breathing of pure oxygen, deficits in arterial oxygenation reflect shunt fraction with minimal influence of low ventilation/perfusion units. A fast responding $\mathrm{PaO}_{2}$ sensor records changes within each breath. These $\mathrm{PaO}_{2}$ oscillations can therefore be used to assess $\mathrm{c}-\mathrm{R} / \mathrm{D}$.

$\mathrm{PaO}_{2}$ was measured at $10-\mathrm{Hz}$ digital sampling frequency in the ascending aorta by an indwelling oxygen-sensing probe (MFPF/ FOXY AL-300, OceanOptics, Dunedin, FL) (13). The probe was

\section{Assessment of Regional Ventilation Distribution by EIT}

To assess regional ventilation distribution, a 16-electrode EIT system (Goe-MF II study device, CareFusion, Hoechberg, Germany) was used. The 16 skin electrodes (ECG electrodes Red Dot 2239, 3M HealthCare, Borken, Germany) were placed on the chest circumference in one transversal lung section, approximately $10 \mathrm{~cm}$ cranial to the diaphragm. A reference electrode was placed $4 \mathrm{~cm}$ below the acquisition plane. EIT data were acquired applying an electrical current of $5 \mathrm{~mA}$ at $50 \mathrm{kHz}$. Voltage differences between neighboring electrode pairs were measured in a sequential rotating process which obtained $13 \mathrm{~Hz}$. Each recording lasted for 2 minutes. Functional EIT scans were generated offline using AUSPEX software 


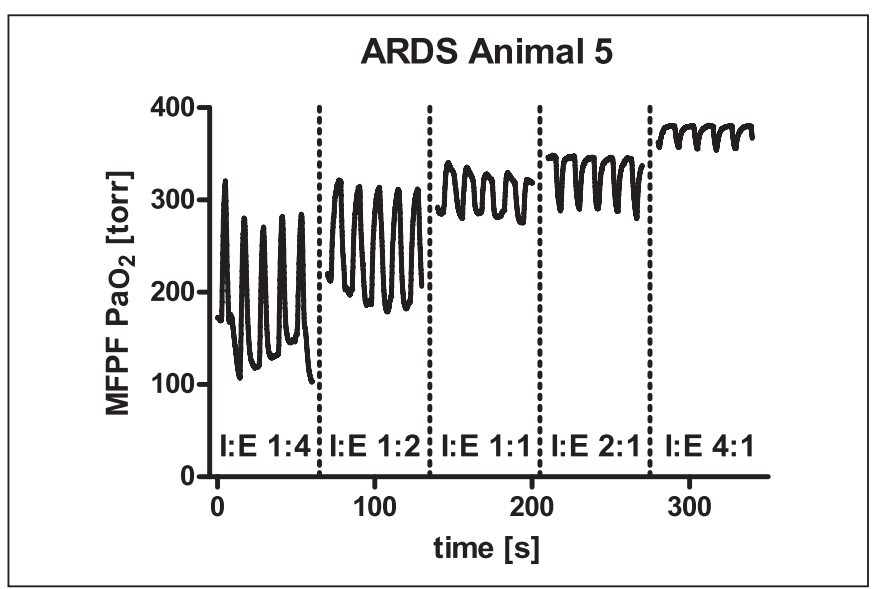

Figure 2. Example of multifrequency phase fluorimetry (MFPF) raw data (animal 5) in experimental acute respiratory distress syndrome (ARDS) showing the $\mathrm{PaO}_{2}$ over time for the different inspiration to expiration (I:E) ratios during ongoing mechanical ventilation.

(v.1.7, Department of Physics and Medical Technology, VU Medical Centre Amsterdam, Amsterdam, The Netherlands) as described previously (14) and further postprocessed to evaluate regional distribution of ventilation. For each scan, the amplitudes of relative impedance changes (rel. $\Delta \mathrm{Z}$ ) were calculated pixel-wise (matrix of $32 \times 32$ ) and normalized to obtain the percentaged distribution of Vт $(15,16)$. Using an established approach, ventrodorsal profiles of regional percentaged Vт distribution were computed for all 32 horizontal layers (17, 18). To present the changes in regional ventilation distribution between the different I:E ratios, regional ventilation difference profiles to I:E 1:4 were generated and the geometrical centers of ventilation (COVs) were assessed (19), representing the location of maximal ventilation within the anteroposterior chest diameter in percent.

\section{Statistical Analysis}

This experimental series was performed to investigate the influence of I:E ratio upon $\triangle \mathrm{PaO}_{2}$ (primary endpoint) and Horowitz index $\left(\mathrm{PaO}_{2} / \mathrm{FIO}_{2}\right)$ (secondary endpoint). As the assumption of normal distribution was not violated (Kolmogorov-Smirnov test), the effects between the five different I:E parameter values of 1:4, 1:2, 1:1, 2:1, and 4:1 were tested by a mixed analysis of variance (ANOVA) model, which also includes the structure of dependency due to repeated measures. The influence of time was accounted by modeling a heterogeneous, autoregressive process of first order. Randomization list was created prior to the experiments using Research Randomizer software (www.researchrandomizer.org). Thus, we tested whether there is a significant difference in (MFPF$\left.\Delta \mathrm{PaO}_{2}\right)$ and Horowitz index $\left(\mathrm{PaO}_{2} / \mathrm{FIO}_{2}\right)$ (dependent variables) with regard to the I:E ratio (fixed variable). We adjusted for multiple testing by the Bonferroni-Holm method. Estimates and $95 \%$ CIs for the tested effects in the mixed model are provided. The multiple tests performed keep a multiple level of $\alpha=5 \%$. Influence of mean airway pressure $\left(\mathrm{M}_{\mathrm{PAW}}\right)$ upon MFPF- $\triangle \mathrm{PaO}_{2}$ was assessed considering the correlation within the subjects (20).

Changes in regional ventilation distribution were evaluated by assessing the ventrodorsal profiles, the ventrodorsal difference
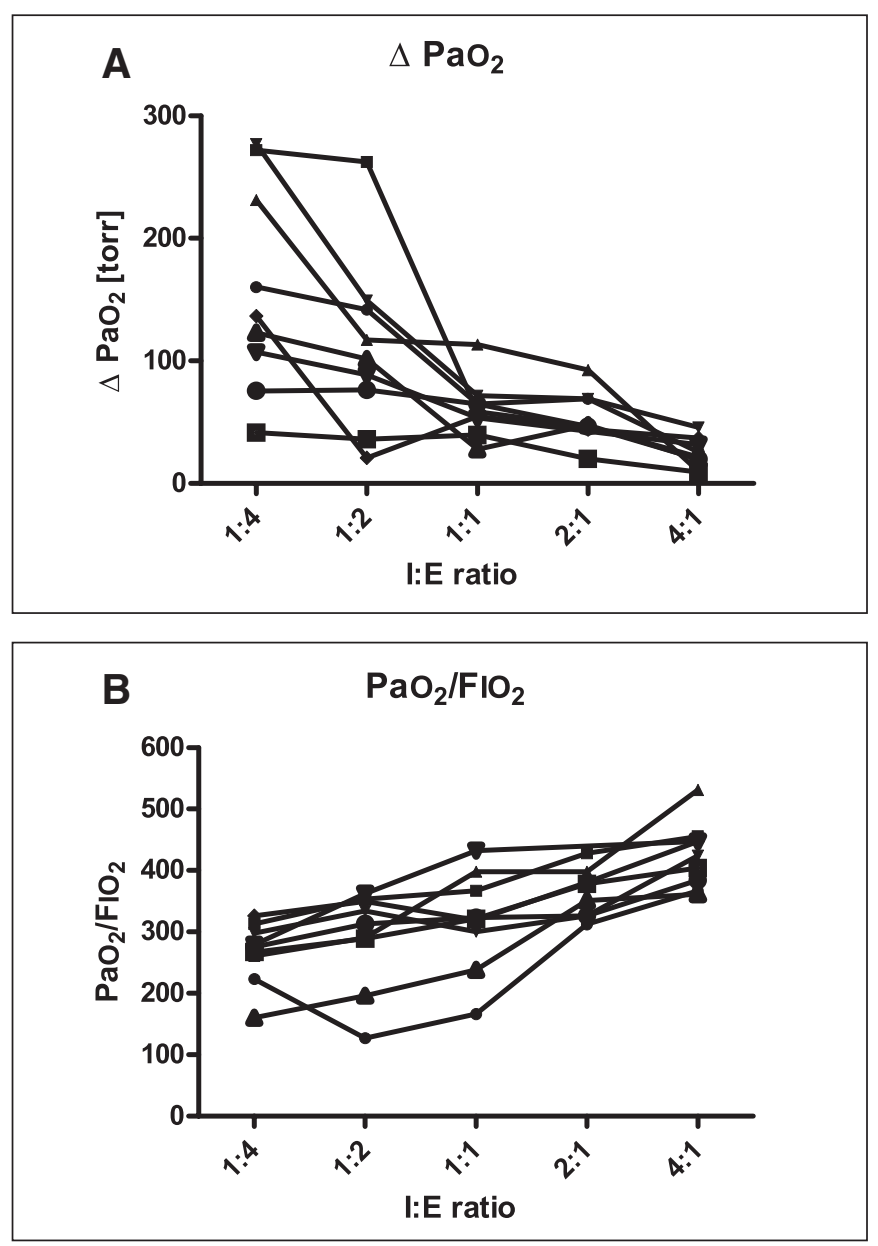

Figure 3. Itemized for each study subject, the scatter plots show the behavior of multifrequency phase fluorimetry- $\Delta \mathrm{PaO}_{2}(\mathbf{A})$ and of Horowitz index $\left(\mathrm{PaO}_{2} / \mathrm{FIO}_{2}\right)(\mathbf{B})$ in accordance with the different randomized inspiration to expiration (I:E) ratios.

profiles to I:E 1:4 and the geometrical COV. To show differences in COV, repeated-measures ANOVA was performed, as the data were normally distributed (Kolmogorov-Smirnov test).

To identify the time constants (TAU) of recruitment and derecruitment, $\mathrm{PaO}_{2}$ versus time data were normalized to obtain the percentaged increase or decrease in $\mathrm{Po}_{2}$ for each animal. For each measurement, nonlinear fitting by extra sum-ofsquare $F$ test was performed to choose between a one-phase or two-phase exponential model. Detailed model description is given in supplemental material (Supplemental Digital Content 1, http://links.lww.com/CCM/B150). Differences in TAU values between recruitment and derecruitment were assessed by Wilcoxon signed rank test. Statistical analyses were performed using SAS (v.9.3, SAS Institute, Cary, NC), SPSS (v.18, SPSS, Chicago, IL), and GraphPad Prism (v.6.0, GraphPad Software, La Jolla, CA).

\section{RESULTS}

\section{MFPF- $\triangle \mathrm{PaO}_{2}$ and Horowitz Index $\left(\mathrm{PaO}_{2} / \mathrm{FIO}_{2}\right)$}

In the $\mathrm{BLH}$ condition, MFPF- $\triangle \mathrm{PaO}_{2}$ ranged between 3 torr $(0.4$ $\mathrm{kPa})$ (I:E 4:1) and 18 torr (2.4 kPa) (I:E 1:4) for all the different 
$\mathrm{I}: \mathrm{E}$ ratios. $\mathrm{PaO}_{2} / \mathrm{FIO}_{2}$ ratio showed values between $636 \pm 49$ (I:E $1: 4)$ and $631 \pm 50$ (I:E 4:1).

After induction of lung injury (3.4 \pm 1.2 lavages with a total volume of $3.2 \pm 1.1 \mathrm{~L}$ normal saline), c-R/D occurred in all subjects, routinely with the highest MFPF- $\triangle \mathrm{PaO}_{2}$ at I:E of $1: 4$ ranging from 52 torr $(6.9 \mathrm{kPa})$ to 277 torr $(36.9 \mathrm{kPa})$ with a mean of $158 \pm 85$ torr $(21.1 \pm 11.3 \mathrm{kPa})$ at the predefined ventilator settings. Under these conditions, Horowitz index $\left(\mathrm{PaO}_{2} / \mathrm{FIO}_{2}\right)$ decreased to $253 \pm 32$.
MFPF- $\Delta \mathrm{PaO}_{2}$ and $\mathrm{PaO}_{2} / \mathrm{FIO}_{2}$ as a Function of I:E Ratio in Lavage Injury

In the model ARDS setting, IRV ventilation led to a significant decrease in c-R/D and to an increase in $\mathrm{PaO}_{2} / \mathrm{FIO}_{2}$. An example (animal 5) is given in Figure 2. As investigated by variation of I:E ratio, mean MFPF- $\triangle \mathrm{PaO}_{2}$ decreased from $158 \pm 85$ torr $(21.1 \pm 11.3 \mathrm{kPa})(\mathrm{I}: \mathrm{E} 1: 4)$ to values of $25 \pm 12$ torr $(3.3 \pm 1.6 \mathrm{kPa})(\mathrm{I}: \mathrm{E} 4: 1)$, whereas mean $\mathrm{PaO}_{2} / \mathrm{FIO}_{2}$ increased from $267 \pm 50$ (I:E 1:4) to $424 \pm 53$ (I:E 4:1). Intrinsic PEEP

\section{TABLE 1. Showing the Estimates and $95 \%$ Cls of the Mixed Analysis of Variance Model Analysis for the Defined Main Endpoints Multifrequency Phase Fluorimetry $\Delta \mathrm{PaO}_{2}$ and the Horowitz Index $\left(\mathrm{PaO}_{2} / \mathrm{FIO}_{2}\right)$}

\begin{tabular}{|c|c|c|c|}
\hline \multirow[b]{2}{*}{ I:E Ratio } & \multirow[b]{2}{*}{ Estimates } & \multicolumn{2}{|c|}{$95 \% \mathrm{Cl}$} \\
\hline & & Lower Bound & Upper Bound \\
\hline \multicolumn{4}{|c|}{ Multifrequency phase fluorimetry $\Delta \mathrm{PaO}_{2} \pm \mathrm{SE}($ torr $[\mathrm{kPa}])$} \\
\hline $\mathrm{I}: \mathrm{E} 1: 4$ & $158.3 \pm 17.3(21.1 \pm 2.3)$ & $123.4(16.5)$ & $193.2(25.8)$ \\
\hline $\mathrm{I}: \mathrm{E} 1: 2$ & $113.0 \pm 17.3(15.1 \pm 2.3)$ & $78.2(10.4)$ & $147.9(19.7)$ \\
\hline $\mathrm{I}: \mathrm{E} 1: 1$ & $61.0 \pm 17.3(8.1 \pm 2.3)$ & $26.2(3.5)$ & $95.9(12.8)$ \\
\hline $\mathrm{I}: \mathrm{E} 2: 1$ & $51.6 \pm 18.3(6.9 \pm 2.4)$ & $14.6(1.9)$ & $88.6(11.8)$ \\
\hline I:E 4:1 & $24.6 \pm 17.3(3.3 \pm 2.3)$ & $-10.3(-1.4)$ & $59.5(7.9)$ \\
\hline \multicolumn{4}{|c|}{$\mathrm{PaO}_{2} / \mathrm{FlO}_{2} \pm \mathrm{SE}$} \\
\hline $\mathrm{I}: \mathrm{E} 1: 4$ & $266.9 \pm 21.1$ & 224.2 & 309.6 \\
\hline l:E 1:2 & $290.4 \pm 21.1$ & 247.7 & 333.1 \\
\hline $\mathrm{I}: \mathrm{E} 1: 1$ & $318.3 \pm 21.1$ & 275.6 & 360.9 \\
\hline $\mathrm{I}: \mathrm{E} 2: 1$ & $362.4 \pm 22.4$ & 317.1 & 407.7 \\
\hline I:E 4:1 & $424.5 \pm 21.1$ & 381.8 & 465.2 \\
\hline
\end{tabular}

$\mathrm{I}: \mathrm{E}=$ inspiration to expiration ratio.

\section{TABLE 2. Results of the Paired Comparisons Performed by Mixed Analysis of Variance Model Analysis}

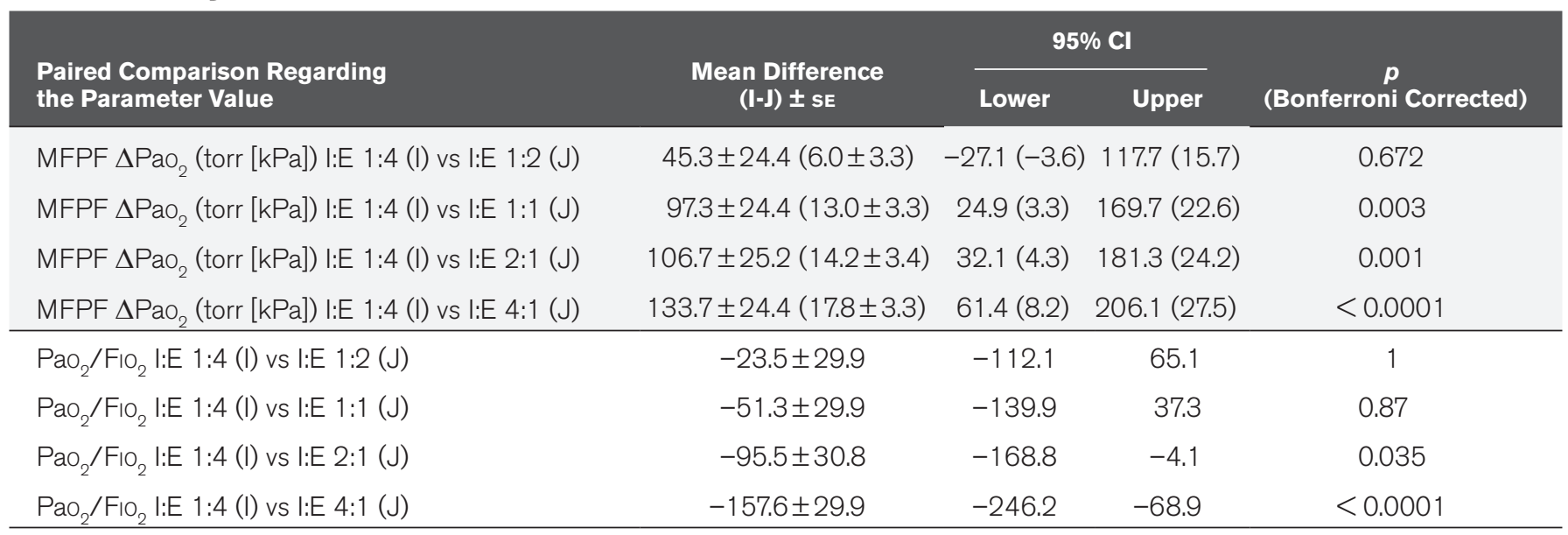

MFPF $=$ multifrequency phase fluorimetry, $\mathrm{I}: \mathrm{E}=$ inspiration to expiration ratio.

Mean differences with SES are given, as well as the $95 \%$ Cls. $p$ values, Bonferroni-Holm corrected for multiple testing. 


\section{TABLE 3. Mean and SD of the Different Spirometry and Hemodynamic Parameter Values}

\begin{tabular}{|c|c|c|c|c|c|c|}
\hline Measurement & $\begin{array}{c}\mathbf{P}_{\text {encfinss }} \\
\left.\text { (cm } \mathrm{H}_{2} \mathbf{6}\right)\end{array}$ & $\begin{array}{c}\mathrm{M}_{\mathrm{PAW}} \\
\left(\mathrm{cm} \mathrm{H_{2 }} \mathbf{O}\right)\end{array}$ & $\begin{array}{l}V_{T_{\mathrm{ex}}} \\
(\mathrm{mL})\end{array}$ & 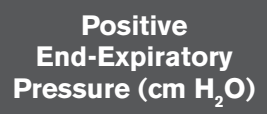 & $\begin{array}{l}\text { Etco } \\
\text { (torr) }\end{array}$ & $\left(\mathrm{mL} / \mathrm{cm}_{\mathrm{dyn}} \mathrm{H}_{2} \mathrm{O}\right)$ \\
\hline Baseline & $40 \pm 6$ & $10 \pm 3$ & $672 \pm 160$ & $5 \pm 0.3$ & $37 \pm 3$ & $26 \pm 5$ \\
\hline ARDS I:E 1:4 & $39 \pm 10$ & $13 \pm 4$ & $573 \pm 178$ & $5 \pm 0.3$ & $39 \pm 3$ & $18 \pm 3$ \\
\hline ARDS I:E 1:2 & $40 \pm 7$ & $18 \pm 3$ & $558 \pm 258$ & $5 \pm 0.3$ & $38 \pm 3$ & $20 \pm 5$ \\
\hline ARDS I:E 1:1 & $40 \pm 7$ & $24 \pm 3$ & $520 \pm 173$ & $5 \pm 0.3$ & $37 \pm 4$ & $21 \pm 4$ \\
\hline ARDS I:E 2:1 & $40 \pm 6$ & $29 \pm 4$ & $526 \pm 167$ & $5 \pm 0.3$ & $37 \pm 3$ & $21 \pm 4$ \\
\hline ARDS I:E 4:1 & $41 \pm 6$ & $31 \pm 4$ & $600 \pm 152$ & $5 \pm 0.4$ & $39 \pm 4$ & $21 \pm 4$ \\
\hline
\end{tabular}

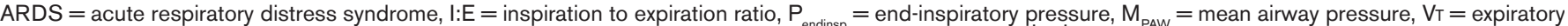
tidal volume, $\mathrm{Etco}_{2}=$ end-tidal carbon dioxide concentration, $\mathrm{C}_{\mathrm{dyn}}=$ compliance of the respiratory system, $\dot{Q}_{s} / Q_{t}=$ calculated intrapulmonary shunt.

was measured to be less than $0.5 \mathrm{~cm} \mathrm{H}_{2} \mathrm{O}$ at all settings. The scatter plots (Fig. 3) show the detailed individual results of each subject for the five different I:E ratios. Estimates with $95 \% \mathrm{CI}$ and the pairwise comparisons of the mixed linear model are presented in Tables 1 and 2. Additionally, Table 3 shows mean and SD of spirometry and hemodynamic parameters. To further investigate the dependency of $\Delta \mathrm{PaO}_{2}$, on mean airway pressure $\left(\mathrm{M}_{\mathrm{PAW}}\right)$, a correlation coefficient with repeated observations was calculated showing an $R^{2}$ of 0.58 .

\section{Regional Ventilation Distribution}

In healthy lungs, I:E variation led to no relevant changes in distribution of ventilation. In model ARDS, the variation of the I:E ratio led to different regional ventilation distribution, as observed by EIT. Titrating the I:E ratio in direction of IRV was accompanied by redistribution of ventilation from nondependent toward dependent lung regions
(Fig. 4). COV analysis reflected this finding, as the COV shifted to dorsal lung regions when shortening exhalation time (Fig. 5).

\section{Time Constants for Recruitment and Derecruitment by MPFP-Po,}

To further learn about the temporal behavior of $\mathrm{c}-\mathrm{R} / \mathrm{D}$, time constants of recruitment and derecruitment were evaluated. For all animals, the extra sum-of-square $F$ test showed that the two-phase exponential model was superior to a onephase exponential model, for both recruitment and derecruitment. An example (animal 5) of raw data time course with the fitted model appears in Figure 6, and the complete set of curve fits, as well as the detailed results of extra sumof-square $F$ test, is available as supplemental material (Supplemental Digital Content 1, http://links.lww.com/CCM/ B150). Overall, lung opening showed that the injured lungs

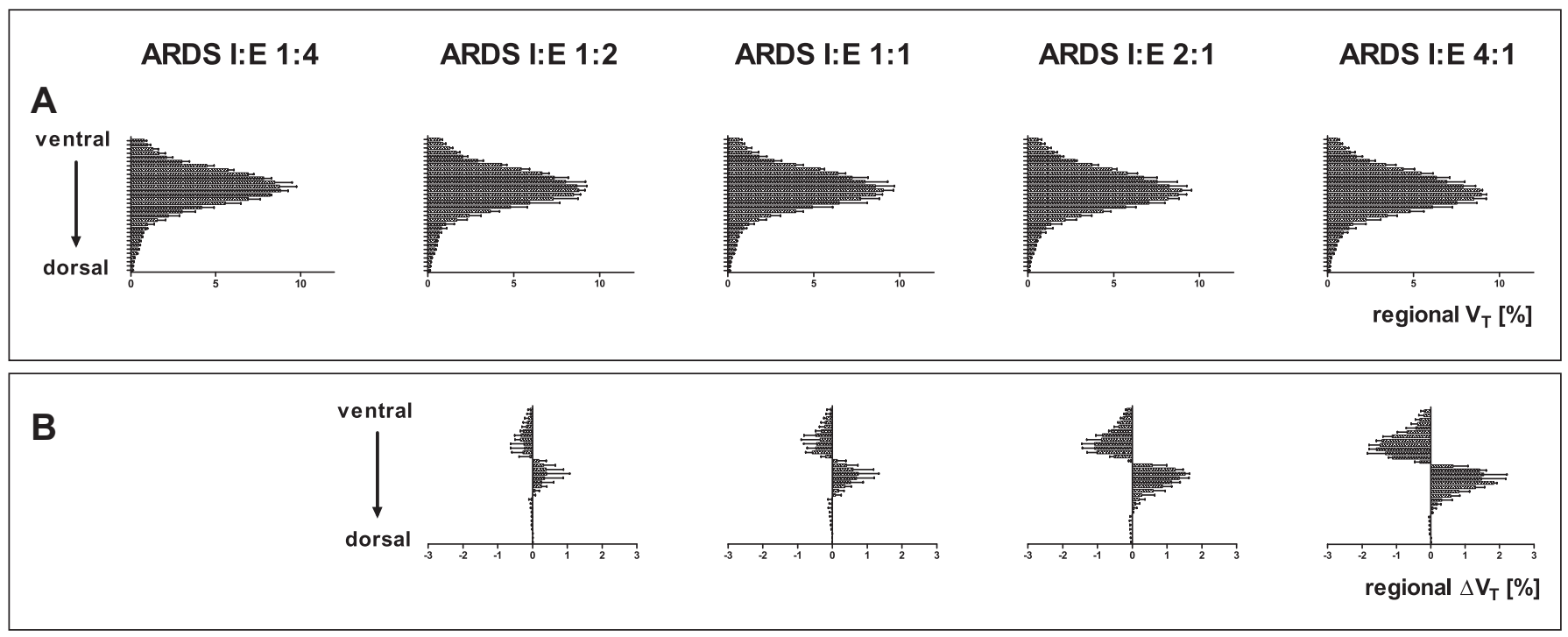

Figure 4. Regional ventilation distribution as assessed by electrical impedance tomography. A, Regional ventilation distribution of tidal volume (VT) in percent is shown for 32 horizontal layers from ventral to dorsal within the chest cross-section (ventrodorsal profiles). Bars represent the median, whiskers the 95\% $\mathrm{Cl}$ of nine animals in mild acute respiratory distress syndrome (ARDS), itemized for the different inspiration to expiration (I:E) ratios. B, Differences in regional ventilation distribution of $\mathrm{VT}$ as compared with I:E 1:4 $(\Delta \mathrm{V} T)$ are presented for 32 horizontal layers from ventral to dorsal within the chest cross-section (difference ventrodorsal profiles). Bars represent the median, whiskers the $95 \% \mathrm{Cl}$ of nine animals in mild ARDS, itemized for the different I:E ratios. 


\begin{tabular}{cccccc}
$\begin{array}{c}\text { Heart Rate } \\
(\mathbf{b p m})\end{array}$ & $\begin{array}{c}\text { Mean Arterial } \\
\text { Pressure } \\
(\mathbf{m m} \mathbf{~ H g})\end{array}$ & $\begin{array}{c}\text { Central } \\
\text { Venous Pressure } \\
(\mathbf{m b a r})\end{array}$ & $\begin{array}{c}\text { Cardiac } \\
\text { Output } \\
(\mathbf{L} / \mathbf{m i n})\end{array}$ & $\begin{array}{c}\text { Mean Pulmonary } \\
\text { Arterial Pressure } \\
(\mathbf{m m} \mathbf{~ H g})\end{array}$ & $\begin{array}{c}\mathbf{\mathbf { a }}_{\mathbf{s}} / \dot{\mathbf{a}}_{\mathbf{t}} \\
(\%)\end{array}$ \\
\hline $101 \pm 16$ & $88 \pm 16$ & $8 \pm 1$ & $4.2 \pm 2.3$ & $17 \pm 3$ & $4 \pm 1$ \\
$121 \pm 30$ & $79 \pm 12$ & $9 \pm 3$ & $4.8 \pm 1.8$ & $32 \pm 3$ & $20 \pm 5$ \\
$122 \pm 34$ & $74 \pm 20$ & $10 \pm 3$ & $4.5 \pm 1.9$ & $31 \pm 4$ & $23 \pm 12$ \\
$121 \pm 34$ & $70 \pm 18$ & $11 \pm 4$ & $4.7 \pm 2.1$ & $34 \pm 3$ & $16 \pm 5$ \\
$121 \pm 33$ & $66 \pm 14$ & $11 \pm 5$ & $4.2 \pm 1.8$ & $35 \pm 4$ & $11 \pm 3$ \\
$125 \pm 29$ & $65 \pm 13$ & $12 \pm 4$ & $4.3 \pm 1.9$ & $36 \pm 3$ & $9 \pm 3$ \\
\hline
\end{tabular}

were fast to recruit, whereas derecruitment in contrast was found to be slower ( $p=0.0078$ ). Table 4 summarizes the time constants of all animals.

\section{DISCUSSION}

The present study focused on comparing the effects of different I:E ratios upon respiratory-dependent fluctuations in $\mathrm{PaO}_{2}$ and upon Horowitz index. Our findings demonstrate that diminished exhalation time by IRV is associated with a significant reduction of $\Delta \mathrm{PaO}_{2}$ while maintaining end-expiratory recruitment at high levels. These findings were not an effect of intrinsic PEEP. The temporal dynamics of collapse and reopening of atelectasis can explain this behavior. In this saline lavage model of ARDS, lung tissue was fast to recruit while derecruitment was the more time-consuming process. As a consequence, shortening the exhalation time with IRV

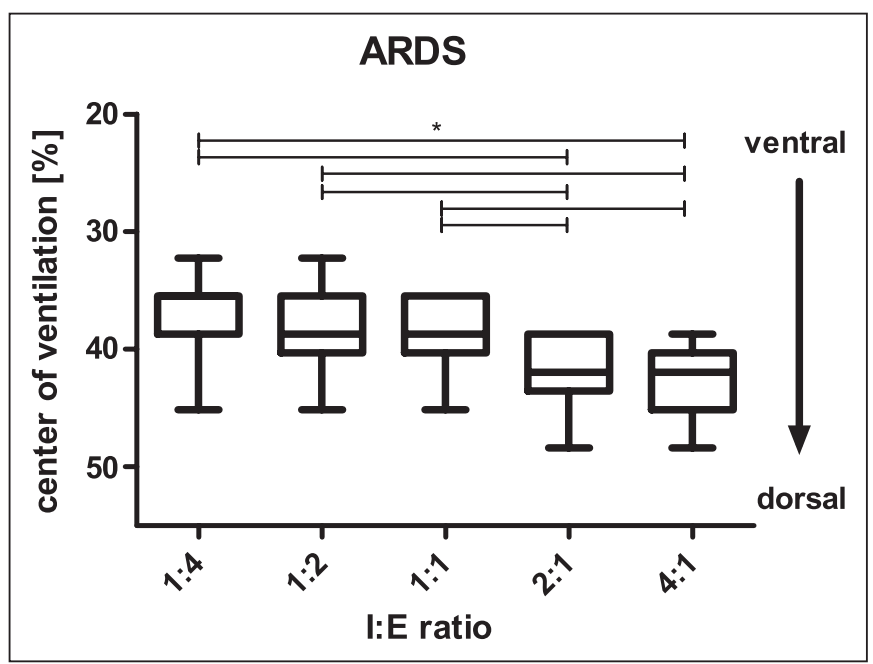

Figure 5. Center of ventilation (COV) as assessed by electrical impedance tomography. Data present the geometrical COV in percent of the chest diameter from ventral to dorsal. Box plots represent nine animals in mild acute respiratory distress syndrome (ARDS) for the different inspiration to expiration (I:E) ratios. Boxes show the median, the 25th and the 75th percentile, whiskers the 10\% and 90\% Cl. To describe the differences between the I:E ratios, repeated-measures ANOVA was assessed, where asterisk indicates a $p<0.05$. reduced the time available to derecruit, resulting in more average recruitment (as assessed by the $\mathrm{PaO}_{2} / \mathrm{FIO}_{2}$ ratio) and less cyclic recruitment (as assessed by $\Delta \mathrm{PaO}_{2}$ ). The redistribution of regional ventilation from nondependent toward dependent lung regions during IRV is consistent with these findings.

We chose the widely used surfactant depletion model to induce a mild degree of injury (21) to examine the timedependent characteristics of atelectasis and alveolar recruitment $(22,23)$. The main intent was to induce large amounts of recruitable but minimal fixed atelectasis to investigate $c-R / D$. Therefore, a novel standardized routine was implemented to titrate a mild lung damage and not to produce a model that fulfilled Horowitz index criteria for ARDS. Our results demonstrate that $\mathrm{c}-\mathrm{R} / \mathrm{D}$ could be induced in all subjects by the performed model, as measured by real-time $\mathrm{PaO}_{2}$ sensing. The MFPF method we used was suitable to detect $\mathrm{PaO}_{2}$ at high temporal resolution and previously showed excellent agreement with standard Clark-type electrode-based blood gas $\mathrm{PO}_{2}$ measurements $\left(R^{2}, 0.99\right)(13)$.

Neumann et al (24) and then Markstaller et al (25) both showed, by using dCT to follow responses to step changes in airway pressure, that the time constants for recruitment and derecruitment in saline lavage are on the order of a breath cycle . Baumgardner et al (12) reported huge respirationdependent fluctuations in $\mathrm{PaO}_{2}$ in saline-lavaged rabbits that could be attributed to $c-R / D$. As several studies indicate more severe damage during $\mathrm{c}-\mathrm{R} / \mathrm{D}$ and a higher inflammatory response in lung areas where c-R/D occurs $(26,27)$, novel ventilatory strategies are recently under discussion to avoid $\mathrm{c}-\mathrm{R} / \mathrm{D}$ during mechanical ventilation. In general, a major principle of lung-protective ventilation strategies is to use small VTs s to prevent further VILI $(3,28,29)$. On the other hand, low VT ventilation can lead to formation of atelectasis and to an increase in intrapulmonary shunt fraction. Although several studies clearly demonstrated the advantages of lower VTs, the specific role of atelectrauma in VILI in clinical patients is less clear.

High PEEP levels are commonly used to maintain recruitment by higher end-expiratory alveolar pressures, but possibly 


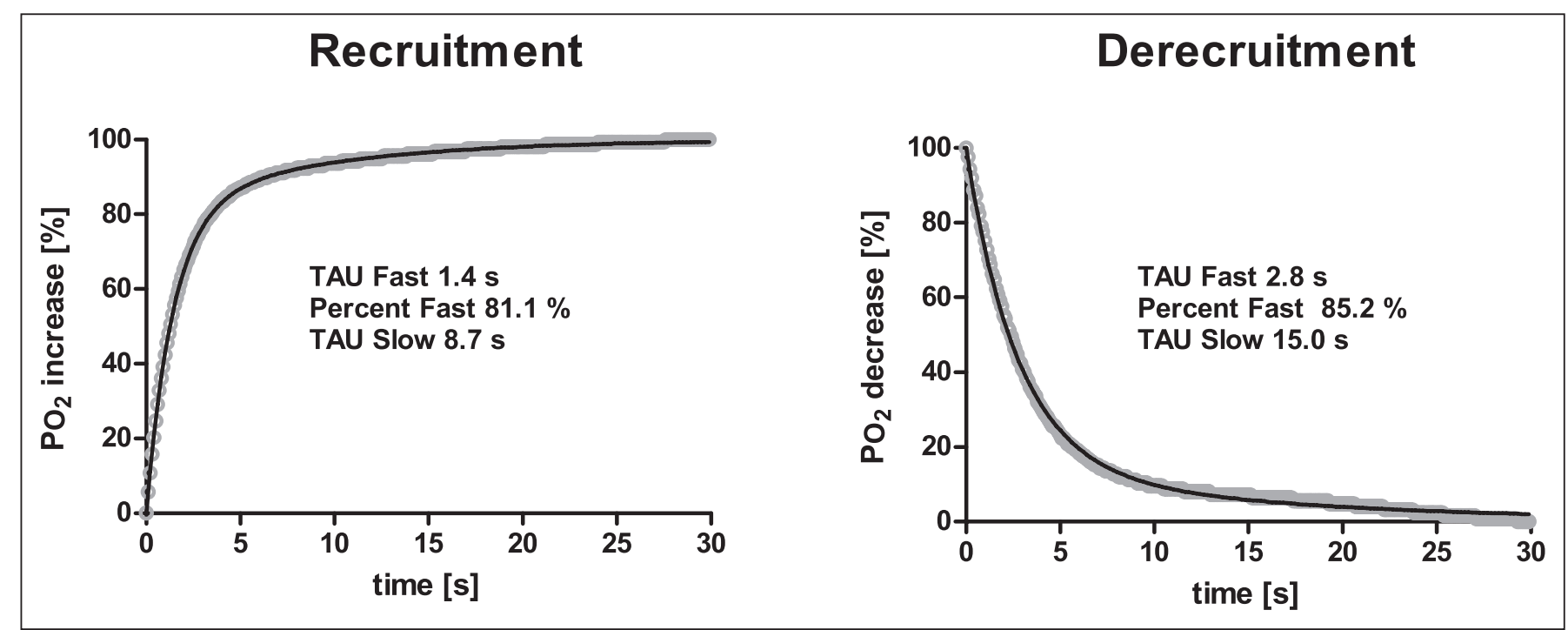

Figure 6. Example of time constant assessment. Showing the measured $\mathrm{Po}_{2}$ in percent over time during step change in airway pressure (gray circles) with the corresponding two-phase exponential model fit (dark line). Left, For recruitment by step change in airway pressure from 5 to $40 \mathrm{~cm} \mathrm{H}_{2} \mathrm{O}$. Right, For derecruitment by step change in airway pressure from 40 to $5 \mathrm{~cm} \mathrm{H}_{2} \mathrm{O}$.

TABLE 4. Assessment of Time Constants for Recruitment and Derecruitment

\begin{tabular}{|c|c|c|c|c|c|c|}
\hline \multirow[b]{3}{*}{ Animal } & \multicolumn{6}{|c|}{ Two-Phase Exponential Model Fit } \\
\hline & \multicolumn{3}{|c|}{ Recruitment } & \multicolumn{3}{|c|}{ Derecruitment } \\
\hline & TAU Fast (s) & Percent Fast & TAU SIow (s) & TAU Fast (s) & Percent Fast & TAU Slow (s) \\
\hline 1 & 1.4 & 76.6 & 8.6 & 2.1 & 91.9 & 19.1 \\
\hline 2 & 1.4 & 81.0 & 8.7 & 4.3 & 78.7 & 16.5 \\
\hline 3 & 1.4 & 79.8 & 9.7 & 3.0 & 88.9 & 16.8 \\
\hline 4 & 1.7 & 79.5 & 10.2 & 2.9 & 90.2 & 19.0 \\
\hline 5 & 1.4 & 81.0 & 8.7 & 2.8 & 85.2 & 15.0 \\
\hline 6 & 2.4 & 73.5 & 9.2 & 4.4 & 79.1 & 16.7 \\
\hline 7 & 1.6 & 73.8 & 9.7 & 2.9 & 91.7 & 23.1 \\
\hline 8 & 1.6 & 78.1 & 8.9 & 2.2 & 88.5 & 15.4 \\
\hline Average & 1.6 & 77.9 & 9.2 & 3.1 & 86.8 & 17.7 \\
\hline
\end{tabular}

Percent fast is the fraction in percent accounted for by the faster of the two components. TAU fast and TAU slow are the two time constants in seconds.

at the cost of increased lung distention and reduced cardiac output (30-32). We hypothesized that IRV might be used to maintain end-expiratory recruitment without the adverse effects of PEEP. To investigate purely the influence of I:E ratio, we provoked maximal c-R/D at fixed ventilator settings for all measurements, and then randomly varied only the I:E ratio. Due to the standardization of $\mathrm{P}_{\text {endinsp }}$ in PCV mode, $\mathrm{VT}$ could not be controlled; however, we observed only slight differences in $\mathrm{V}_{\text {T }}$ between the different I:E ratios. The slow RR was chosen to provoke maximal c-R/D and not to simulate clinical practice. For the direct comparison of $\Delta \mathrm{PaO}_{2}$ between the different I:E ratios, we used an $\mathrm{FiO}_{2}$ of 1.0 for all measurements. Thus, all measured $\mathrm{PaO}_{2}$ values were above 90 torr $(12 \mathrm{kPa})$ and therefore in the saturated part of the hemoglobin dissociation curve. Hence, our ventilator settings were designed to optimize the study of our main interest, the effect of I:E ratios on c-R/D, and not to mimic protective ventilation strategies.

We further investigated the time course of $\mathrm{PaO}_{2}$ changes during lung opening (recruitment) (CPAP 5 to $40 \mathrm{~cm} \mathrm{H}_{2} \mathrm{O}$ ) and collapse (derecruitment) (CPAP 40 to $5 \mathrm{~cm} \mathrm{H}_{2} \mathrm{O}$ ). Our results suggest that in saline-lavaged pigs with mild ARDS, derecruitment is a slower process than recruitment. This is in line with the findings by Markstaller et al (25), who calculated similar time constants by dCT for a CPAP maneuver from 0 to $40 \mathrm{~cm}_{2} \mathrm{O}$. Also, using intravital microscopy, Albert et al (33) observed about $50 \%$ of recruitment in the first 2 seconds after a $30 \mathrm{~cm} \mathrm{H}_{2} \mathrm{O}$ recruitment maneuver, in saline-lavaged rat lungs. 
Our study was focused on examining the difference in recruitment and derecruitment dynamics as the mechanism for effects of I:E ratio on $\Delta \mathrm{PaO}_{2}$ and was not designed to directly address the overall effect of I:E ratio on VILI in clinical or experimental ARDS. The ventilation pattern was intended to provoke maximal $\Delta \mathrm{PaO}_{2}$ for this investigation and clearly does not reflect typical clinical practice. Additionally, the time constants for recruitment and derecruitment in ARDS patients in response to step changes in airway pressure have not been reported. The effects of lung size, species, type of lung injury, and features of lung mechanics such as collateral ventilation and lung compliance are not currently known. Syring et al, for example, reported maximal $\mathrm{PaO}_{2}$ oscillations in lavaged rabbits at an I:E ratio of 2:1, suggesting that in that model derecruitment was slightly faster than recruitment. The general principle that shortening exhalation times can prevent end-expiratory collapse without an increase in intrinsic PEEP, however, was also demonstrated in the study by Syringe et al (34), in that study by increasing RR. Other potential mechanisms of VILI that could accompany IRV, such as higher static stretch and higher mean airway pressures, possibly resulting in increased inflammation (35-38), were not addressed in our study. IRV also carries some risk of increasing intrinsic PEEP, if not carefully monitored. Finally, the use of IRV to achieve high $\mathrm{PaO}_{2} / \mathrm{FIO}_{2}$ ratios in clinical practice would likely require deep sedation and/or muscle relaxation. Further studies with direct assessment of lung injury will be required to address the overall impact of IRV on VILI. Therefore, we need to caution the use of these experimental findings in clinical practice before we see clinical studies demonstrating safety and feasibility of reducing c-R/D by IRV.

\section{CONCLUSIONS}

In a lavage model of ARDS in juvenile pigs, tidal oscillations in $\mathrm{PaO}_{2}\left(\Delta \mathrm{PaO}_{2}\right)$ can be significantly reduced by IRV, applied to the ventilator settings used for this study. During tidal ventilation, a prolonged inspiratory phase leads to higher average $\mathrm{PaO}_{2}$ while the shortened exhalation time reduces $\Delta \mathrm{PaO}_{2}$, suggesting a reduction in c-R/D with IRV. This behavior is consistent with the time constants for recruitment and derecruitment as assessed by $\mathrm{PaO}_{2}$ responses to step changes in airway pressure where derecruitment was found to be the more time-consuming process. The changes in regional ventilation distribution as I:E ratio varied from 1:4 to 4:1 support this explanation. These experimental findings of beneficial effects on $c-R / D$ by IRV warrant further clinical studies before we can safely translate these findings into clinical practice.

\section{ACKNOWLEDGMENT}

We thank the provision of facilities and equipment by the Department of Anesthesiology, Medical Center of the Johannes-Gutenberg University Mainz, Mainz, Germany.

\section{REFERENCES}

1. de Prost N, Saumon G, Dreyfuss D: Modeling the time-course of ventilator-induced lung injury: What can we learn from interspecies discrepancies? Intensive Care Med 2011; 37:1901-1903
2. Ventilation with lower tidal volumes as compared with traditional tidal volumes for acute lung injury and the acute respiratory distress syndrome. The Acute Respiratory Distress Syndrome Network. N Engl J Med 2000; 342:1301-1308

3. Serpa Neto A, Cardoso SO, Manetta JA, et al: Association between use of lung-protective ventilation with lower tidal volumes and clinical outcomes among patients without acute respiratory distress syndrome: A meta-analysis. JAMA 2012; 308:1651-1659

4. Yilmaz M, Keegan MT, Iscimen $\mathrm{R}$, et al: Toward the prevention of acute lung injury: Protocol-guided limitation of large tidal volume ventilation and inappropriate transfusion. Crit Care Med 2007; 35:1660-1666; quiz 1667

5. Grasso S, Stripoli T, Sacchi M, et al: Inhomogeneity of lung parenchyma during the open lung strategy: A computed tomography scan study. Am J Respir Crit Care Med 2009; 180:415-423

6. Spieth PM, Gama de Abreu M: Lung recruitment in ARDS: We are still confused, but on a higher PEEP level. Crit Care 2012; 16:108

7. Carvalho AR, Pacheco SA, de Souza Rocha PV, et al: Detection of tidal recruitment/overdistension in lung-healthy mechanically ventilated patients under general anesthesia. Anesth Analg 2013; 116:677-684

8. Caironi P, Cressoni M, Chiumello D, et al: Lung opening and closing during ventilation of acute respiratory distress syndrome. Am J Respir Crit Care Med 2010; 181:578-586

9. David M, Karmrodt J, Bletz C, et al: Analysis of atelectasis, ventilated, and hyperinflated lung during mechanical ventilation by dynamic CT. Chest 2005; 128:3757-3770

10. Vena A, Rylander C, Perchiazzi G, et al: Lung sound analysis correlates to injury and recruitment as identified by computed tomography: An experimental study. Intensive Care Med 2011; 37:1378-1383

11. Hartmann EK, Boehme S, Bentley A, et al: Influence of respiratory rate and end-expiratory pressure variation on cyclic alveolar recruitment in an experimental lung injury model. Crit Care 2012; 16:R8

12. Baumgardner JE, Markstaller K, Pfeiffer B, et al: Effects of respiratory rate, plateau pressure, and positive end-expiratory pressure on $\mathrm{PaO}_{2}$ oscillations after saline lavage. Am J Respir Crit Care Med 2002; 166:1556-1562

13. Boehme S, Duenges B, Klein KU, et al: Multi frequency phase fluorimetry (MFPF) for oxygen partial pressure measurement: Ex vivo validation by polarographic clark-type electrode. PLoS One 2013; 8:e60591

14. Frerichs I, Hahn G, Hellige G: Thoracic electrical impedance tomographic measurements during volume controlled ventilation-effects of tidal volume and positive end-expiratory pressure. IEEE Trans Med Imaging 1999; 18:764-773

15. Shi C, Boehme S, Bentley $\mathrm{AH}$, et al: Assessment of regional ventilation distribution: Comparison of vibration response imaging (VRI) with electrical impedance tomography (EIT). PLoS One 2014; 9:e86638

16. Frerichs I, Hahn G, Golisch W, et al: Monitoring perioperative changes in distribution of pulmonary ventilation by functional electrical impedance tomography. Acta Anaesthesio/ Scand 1998; 42:721-726

17. Frerichs I, Schmitz G, Pulletz S, et al: Reproducibility of regional lung ventilation distribution determined by electrical impedance tomography during mechanical ventilation. Physiol Meas 2007; 28:S261-S267

18. Frerichs I, Hahn G, Schröder T, et al: Electrical impedance tomography in monitoring experimental lung injury. Intensive Care Med 1998; 24:829-836

19. Zick G, Elke G, Becher T, et al: Effect of PEEP and tidal volume on ventilation distribution and end-expiratory lung volume: A prospective experimental animal and pilot clinical study. PLoS One 2013; 8:e72675

20. Bland JM, Altman DG: Calculating correlation coefficients with repeated observations: Part $1-$ Correlation within subjects. BMJ $1995 ; 310: 446$

21. Kuckelt W, Dauberschmidt R, Bender V, et al: Experimental investigations in adult respiratory distress syndrome (ARDS). Repeated pulmonary lavage in LEWE-mini-pigs. I. Pulmonary mechanics, gas exchange, and pulmonary hemodynamics. Exp Pathol 1981; 20:88-104 
22. Karmrodt J, Bletz C, Yuan S, et al: Quantification of atelectatic lung volumes in two different porcine models of ARDS. $\mathrm{Br} J$ Anaesth 2006; 97:883-895

23. Wang HM, Bodenstein M, Duenges B, et al: Ventilator-associated lung injury superposed to oleic acid infusion or surfactant depletion: Histopathological characteristics of two porcine models of acute lung injury. Eur Surg Res 2010; 45:121-133

24. Neumann P, Berglund JE, Mondéjar EF, et al: Effect of different pressure levels on the dynamics of lung collapse and recruitment in oleic-acid-induced lung injury. Am J Respir Crit Care Med 1998; 158:1636-1643

25. Markstaller K, Eberle B, Kauczor HU, et al: Temporal dynamics of lung aeration determined by dynamic $C T$ in a porcine model of ARDS. Br J Anaesth 2001; 87:459-468

26. Sinclair SE, Chi E, Lin HI, et al: Positive end-expiratory pressure alters the severity and spatial heterogeneity of ventilator-induced lung injury: An argument for cyclical airway collapse. J Crit Care 2009; 24:206-211

27. Otto CM, Markstaller K, Kajikawa O, et al: Spatial and temporal heterogeneity of ventilator-associated lung injury after surfactant depletion. J Appl Physiol (1985) 2008; 104:1485-1494

28. Amato MB, Barbas CS, Medeiros DM, et al: Effect of a protectiveventilation strategy on mortality in the acute respiratory distress syndrome. N Engl J Med 1998; 338:347-354

29. Bruhn A, Bugedo D, Riquelme F, et al: Tidal volume is a major determinant of cyclic recruitment-derecruitment in acute respiratory distress syndrome. Minerva Anestesio/ 2011; 77:418-426
30. Brower RG, Lanken PN, Maclntyre N, et al; National Heart, Lung, and Blood Institute ARDS Clinical Trials Network: Higher versus lower positive end-expiratory pressures in patients with the acute respiratory distress syndrome. N Engl J Med 2004; 351:327-336

31. Grasso S, Fanelli V, Cafarelli A, et al: Effects of high versus low positive end-expiratory pressures in acute respiratory distress syndrome. Am J Respir Crit Care Med 2005; 171:1002-1008

32. Pinsky MR: The hemodynamic consequences of mechanical ventilation: An evolving story. Intensive Care Med 1997; 23:493-503

33. Albert SP, DiRocco J, Allen GB, et al: The role of time and pressure on alveolar recruitment. J App/ Physiol (1985) 2009; 106:757-765

34. Syring RS, Otto CM, Spivack RE, et al: Maintenance of end-expiratory recruitment with increased respiratory rate after saline-lavage lung injury. J Appl Physiol (1985) 2007; 102:331-339

35. Hong CM, Xu DZ, Lu Q, et al: Low tidal volume and high positive endexpiratory pressure mechanical ventilation results in increased inflammation and ventilator-associated lung injury in normal lungs. Anesth Analg 2010; 110:1652-1660

36. Huang Y, Crawford M, Higuita-Castro N, et al: miR-146a regulates mechanotransduction and pressure-induced inflammation in small airway epithelium. FASEB J 2012; 26:3351-3364

37. Wellman TJ, Winkler T, Costa EL, et al: Effect of local tidal lung strain on inflammation in normal and lipopolysaccharide-exposed sheep*. Crit Care Med 2014; 42:e491-e500

38. Chiumello D, Carlesso E, Cadringher $P$, et al: Lung stress and strain during mechanical ventilation for acute respiratory distress syndrome. Am J Respir Crit Care Med 2008; 178:346-355 